It is perhaps worthy of note that in this configuration the $3,3^{\prime}$-hydrogen atoms would not interfere, whereas should the cis-configuration (without a center of symmetry) have been chosen this would not be the case.

A study of the molecular configuration is now under way. It can be stated that the planes whose interferences are strong and which seem well fitted by the trial structure under consideration are of high index.

The writer wishes to thank Profs. G. L. Clark, of the University of Illinois, and J. Turkevich, of Princeton University, for the use of their laboratories. In addition, he wishes to thank Mr J. S. Fritz, of the University of Illinois, who kindly prepared a Debye-Scherrer pattern of high resolution by means of which final values of the unit-cell parameters were obtained.

\section{References}

Clakk, G. L. \& Pickett, L. W. (1931). J. Amer. Chem. Soc. 53, 167.

Dhar, J. (1932). Indian J. Phys. 7, 43.

HengstenberG, J. \& Mark, H. (1929). Z. Krystallogr. $70,283$.

Karle, I. L. \& Brockway, L. O. (1944). J. Amer. Chem. Soc. 66, 1974.

O'Shaughnessy, M. T. \& Rodebush, W. H. (1940). $J$. Amer. Chem. Soc. 62, 2906.

Pickett, L. W., Walter, G. F. \& Firance, H. (1936). J. Amer. Chem. Soc. 58, 2296.

Rodebush, W. H. \& Feldman, 1. (1946). J. Amer. Chem. Soc. 68, 896.

Acta Cryst. (1948). 1, 159

Note sur la cristallographie de la cantharidine, $\mathrm{C}_{10} \mathrm{H}_{12} \mathrm{O}_{4}$. Par J. GarRIdo. Instituto Nacional de Fisica y Química, Serrano 119, Madrid, Espagne

\author{
(Reçu lo 18 mai 1948)
}

La cantharidine, $\mathrm{C}_{10} \mathrm{H}_{12} \mathrm{O}_{4}$, cristallise dans le système orthorhombique; les cristaux employés dans cette étude ont été obtenus par évaporation lente d'une solution de cantharidine 'Kalbaum' dans de l'acetone.

Les formes observées sont, par ordre d'importance: $(100),(010),(011),(210)$. Les angles mesurés au goniomètre conduisent à la relation des axes:

$$
a: b: c=0,88_{3}: 1: 0,53_{9}
$$

d'accord avec les valeurs données par Negri (1889). Le faciès des cristaux étudiés est representé sur Fig. 1.
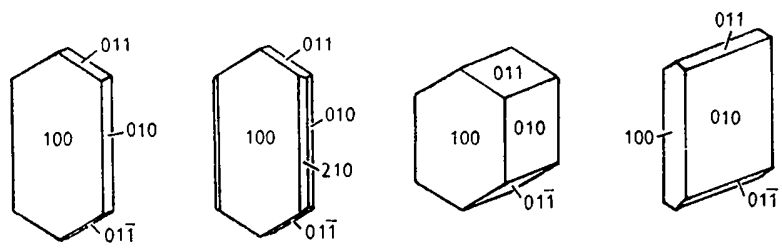

Fig. 1. Le faciès des cristaux de la cantharidine.

J'ai obtenu un certain nombre de diagrammes de Laue, tournants, et de Weissenberg (spectres: $h k 0, h k l$ et $h 0 l$ ). Les dimensions de la maille sont:

$$
a_{0}=11,05 \text { A., } \quad b_{0}=12,54 \text { A. }, \quad c_{0}=6,74 \text { A. }
$$

Une détermination de la densité m'a donné la valeur: 1,41 g.cm..$^{-3}$ Le nombre de molécules par maille est de 4 (densité calculée: 1,38 g.cm. ${ }^{-3}$ ). Les extinctions observées sur les diagrammes sont les suivantes:

$h k 0$-quand $h$ est impair;

$0 k l$-quand $k+l$ est impair;

$h 0 l$-pas d'extinctions.

Ceci donne comme groupe probable le $D_{2 h}^{16}-P n m a$.

La structure de la molécule de la cantharidine a été établie par Gadamer (1904), Diels \& Alder (1929), et récemment par Woodward \& Lotfield (1944). Ces auteurs admettent une formule avec un plan de symétrie. Les conditions de multiplicité du groupe Pnma et la présence de 4 molécules par maille confirment l'éxistence du plan de symétrie des molécules et exigent que celles-ci soient placées dans le cristal de facon à ce que leur plan de symétrie coincide avec le plan $m$ d'orientation (010).

\section{Bibliographie}

Diels, O. \& Alder, K. (1929). Bull. Soc. chim. Fr. 46, 831.

Gadamer, J. (1904). Ber. dtsch. pharm. Ges. 252, 609.

NeGrI, G. V. (1889). Riv. Min. ital. 6, 33.

Woodward, R. B. \& Lotffield, R. I3. (1944). J. Amer. Chem. Soc. 63, 3167.

\title{
Notes and News
}

Announcements and other items of crystallographic interest will be published under this heading at the discretion of the Editorial Board. Copy should be sent direct to the British Co-editor (R. C. Evans, Crystallographic Laboratory, Cavendish Laboratory, Cambridge, England).

\section{International Union of Crystallography}

The adhesion of Great Britain has already been announced in this column (Acta Cryst. (1948) 1, 45). Further formal notification of adhesion has now been given as follows:
On 7 April 1948 by the United States of America through the National Research Council.

On 28 April 1948 by Canada through the National Research Council.

On 31 May 1948 by Norway through the Norwegian Academy of Science. 